\title{
Improving a Solid-State Qubit through an Engineered Mesoscopic Environment
}

\author{
G. Éthier-Majcher, ${ }^{1}$ D. Gangloff, ${ }^{1}$ R. Stockill, ${ }^{1}$ E. Clarke, ${ }^{2}$ M. Hugues,${ }^{3}$ C. Le Gall, ${ }^{1}$ and M. Atatüre ${ }^{1, *}$ \\ ${ }^{1}$ Cavendish Laboratory, University of Cambridge, JJ Thomson Avenue, Cambridge CB3 OHE, United Kingdom \\ ${ }^{2}$ EPSRC National Centre for III-V Technologies, University of Sheffield, Sheffield S1 3JD, United Kingdom \\ ${ }^{3}$ Université Côte d'Azur, CNRS, CRHEA, rue Bernard Gregory, 06560 Valbonne, France
}

(Received 23 June 2017; published 28 September 2017)

\begin{abstract}
A controlled quantum system can alter its environment by feedback, leading to reduced-entropy states of the environment and to improved system coherence. Here, using a quantum-dot electron spin as a control and probe, we prepare the quantum-dot nuclei under the feedback of coherent population trapping and observe their evolution from a thermal to a reduced-entropy state, with the immediate consequence of extended qubit coherence. Via Ramsey interferometry on the electron spin, we directly access the nuclear distribution following its preparation and measure the emergence and decay of correlations within the nuclear ensemble. Under optimal feedback, the inhomogeneous dephasing time of the electron, $T_{2}^{*}$, is extended by an order of magnitude to $39 \mathrm{~ns}$. Our results can be readily exploited in quantum information protocols utilizing spin-photon entanglement and represent a step towards creating quantum many-body states in a mesoscopic nuclear-spin ensemble.
\end{abstract}

DOI: 10.1103/PhysRevLett.119.130503

The interaction between a qubit and its mesoscopic environment offers the opportunity to access and control the ensemble properties of this environment. In turn, tailoring the environment improves qubit performance and can lead to nontrivial collective states. Significant steps towards such control have been taken in systems including nitrogenvacancy centers coupled to ${ }^{13} \mathrm{C}$ spins in diamond [1], superconducting qubits coupled to a microwave reservoir [2], and spins in electrostatically defined [3-5] and selfassembled [6] quantum dots (QDs) coupled to the host nuclei. In InGaAs QDs, the hyperfine interaction permits spin-flip processes to occur between a confined electron and the QD nuclei. Optical pumping of the electron spin induces a directional flipping of nuclear spins leading to a net polarization buildup [7]. The resulting effective magnetic (Overhauser) field can be as strong as $7 \mathrm{~T}$ [8], leading to significant shifts of the electron-spin energy levels [8-11]. In contrast to other systems, the polarization of this isolated mesoscopic ensemble can persist for hours [12]. Coupling the electronic energy shifts to the optical pumping rate closes a feedback loop [13-16] that allows for the selection of the degree of nuclear-spin polarization.

A spectrally sharp version of such stabilizing feedback is achieved through coherent population trapping (CPT), when driving the $\Lambda$ system formed by the two electronspin states and an excited trion state of a negatively charged QD [6,17-20], as depicted in Fig. 1(a). Deviations from the dark-state resonance lead to a preferential driving of one of the two optical transitions, inducing an electron-spin polarization that pulls the Overhauser field back towards a lock point set by the two-photon resonance [Fig. 1(a), bottom panel]. The narrow spectral feature defined by the electronic dark-state coherence thereby carves out a reduced variance Overhauser-field distribution from the initial thermal state with the prospect of improved qubit coherence, as inferred from a number of experiments $[6,21,22]$. However, neither the direct measurement of such a distribution nor of its effect on the electron-spin coherence has been achieved to date. In this Letter, we first prepare optically a reduced-entropy state of the QD nuclear ensemble using CPT-based feedback and then follow its evolution as it interacts with an electron spin in the absence of feedback. We measure the free induction decay (FID) of the qubit and its dephasing time $T_{2}^{*}$ through Ramsey interferometry [23,24]. In this way, we demonstrate that $T_{2}^{*}$ is increased by over an order of magnitude up to tens of nanoseconds. Further, using the qubit coherence as a probe, we observe the emergence and decay of correlations within the tailored nuclear ensemble and thus obtain the time scales characterizing the buildup and decay of available coherence. While Spin Echo offers microsecond coherence $\left(T_{2}\right)$ [23-25], there are many cases such as spin-photon entanglement when it is not applicable, and $T_{2}^{*}$ is thus the relevant time describing the available coherence [26-29]. The extension of $T_{2}^{*}$ well above the trion radiative lifetime presented in this work therefore constitutes a stepping stone towards building quantum networks with quantum dots.

Figure 1(b) displays the experimental sequence used throughout this work. The nuclear ensemble is first prepared by driving the $\Lambda$ system for a time $T_{\mathrm{CPT}}$, followed by $N \sim 100$ consecutive Ramsey interference measurements on the electron spin at a fixed delay of $\tau$ performed during a time $T_{R}$. For this choice of $N$, the Ramsey interferometry does not modify noticeably the prepared nuclear ensemble [16]. Figure 1(c) presents the Ramsey signal measured as a function of $\tau$ in the absence of CPT preparation. There is no fringe visibility at a delay $\tau=$ 42 ns indicating a complete loss of coherence. By contrast, 
(a)

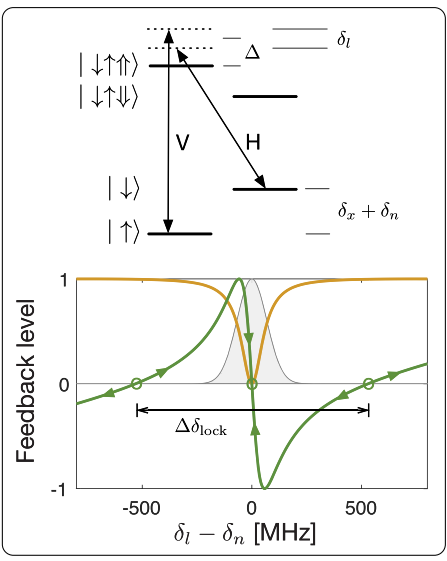

(f)

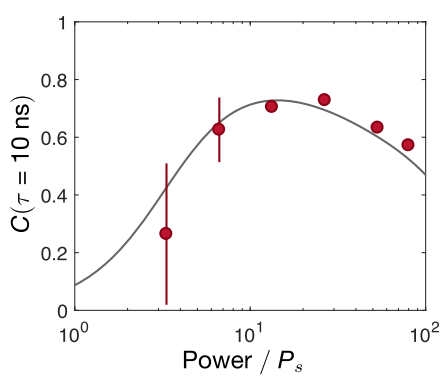

(b)

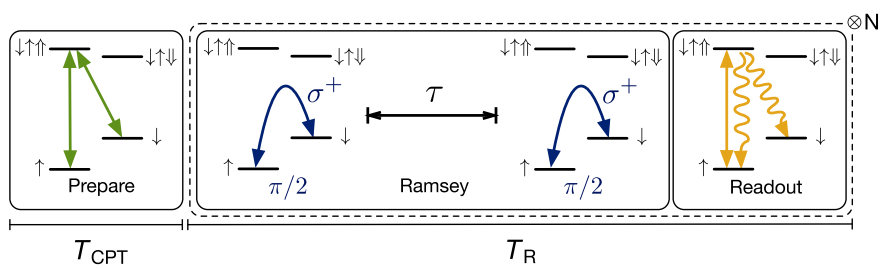

(c)

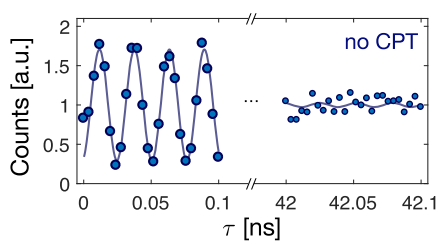

(d)

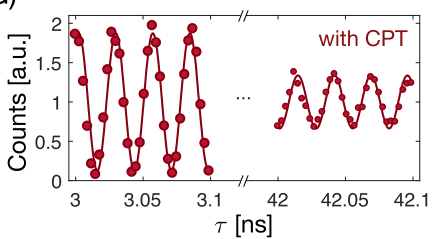

(e)

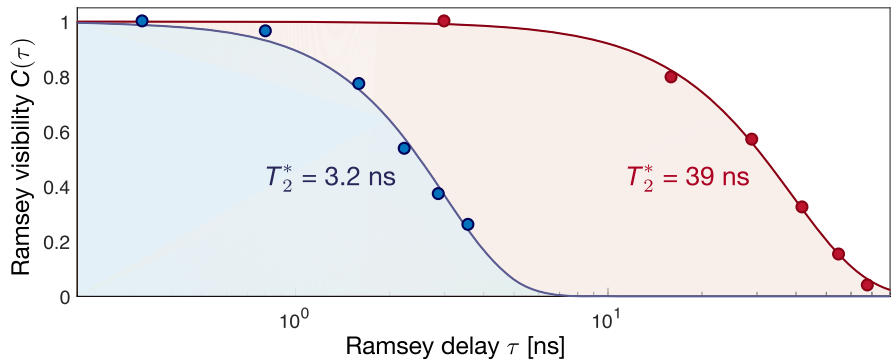

FIG. 1. Extension of the electron $T_{2}^{*}$ via optical preparation of the nuclear ensemble. (a) Top: Energy levels of a singly charged QD in Voigt geometry, driven by two $\sim 965$-nm lasers with single-photon detuning $\Delta>0$ [30] from the excited trion state $\downarrow \uparrow \Uparrow$ and two-photon detuning $\delta_{l}$ ( $H$ and $V$ denote the transition selection rules). The electron-spin splitting between states $\uparrow$ and $\downarrow$ is the sum of the Zeeman splitting $\delta_{x}$ and the Overhauser shift $\delta_{n}$. Bottom: Feedback level set by the ground-state spin polarization (green curve) and normalized scattering rate from the excited state $\Gamma_{h}$ around the dark-state resonance as a function of $\delta_{l}-\delta_{n}$ (yellow curve). $\Delta \delta_{\text {lock }}$ represents the locking range of the feedback mechanism. The Overhauser shift probability distribution from an unprepared nuclear ensemble is shown in gray. (b) Pulse sequence: The nuclear ensemble is prepared by driving the $\Lambda$ system (green arrows) for a time $T_{\text {CPT }}$. Then, $N$ consecutive Ramsey sequences are performed during a time $T_{R}$ : A single sequence consists of two circularly polarized $\pi / 2$ rotation pulses separated by a delay $\tau$, followed by a spin readout performed by driving the high-energy transition and measuring the resonance fluorescence. (c) Ramsey fringes measured with $\mathrm{QD}_{A}$ for an unprepared and (d) prepared nuclear ensemble at $5 \mathrm{~T}$ with $T_{\mathrm{CPT}}=840 \mu \mathrm{s}$ and $T_{R}=210 \mu \mathrm{s}$. (e) Normalized Ramsey visibility as a function of $\tau$ for an unprepared (blue) and prepared (red) ensemble. Solid curves are fitted with $C(\tau)=\exp \left[-\left(\tau / T_{2}^{*}\right)^{\alpha}\right]$, where $T_{2}^{*}=3.2 \pm 0.1 \mathrm{~ns}$ and $\alpha=2.08 \pm 0.04$ in the unprepared case and $T_{2}^{*}=$ $39 \pm 2$ ns and $\alpha=1.9 \pm 0.1$ in the prepared case. (f) Power dependence (relative to the saturation power of a single transition $P_{s}$ ) of the Ramsey visibility at $\tau=10 \mathrm{~ns}$ measured with a different $\mathrm{QD}, \mathrm{QD}_{B}$. The solid curve is calculated from a numerical simulation using a Fokker-Planck formalism.

Fig. 1(d) displays the Ramsey visibility for the same time scales following CPT preparation. We observe that the Ramsey-fringe visibility remains significant at $\tau=42 \mathrm{~ns}$, directly showing a large extension in spin dephasing time due to the ensemble preparation. The dephasing time increases by an order of magnitude from $3.2 \pm 0.1$ to $39 \pm$ 2 ns after CPT feedback [Fig. 1(e)], which unambiguously demonstrates the narrowing of the nuclear-spin distribution. This extension corresponds to reducing the Overhauser-field variance $\sigma^{2} \propto 1 / T_{2}^{* 2}[24,30]$ by $\sim 100$; as a loose comparison, this could be achieved only with net ensemble polarization exceeding 99\% [32].

Qubit coherence is maximal when the width of the darkstate resonance matches the Overhauser-field fluctuations of the unprepared nuclear spins, calculated from the corresponding electron $T_{2}^{*}$ to be $\Delta \delta_{\mathrm{OH}}=160 \pm 12 \mathrm{MHz}$, as represented in Fig. 1(a). The dependence of the Ramseyfringe visibility (at $\tau=10 \mathrm{~ns}$ ) on the optical power shown in Fig. 1(f) indeed reveals the optimal dark-state width to be $\Delta_{\mathrm{CPT}}=163 \pm 19 \mathrm{MHz}$. Lower driving power, corresponding to a narrow dark-state resonance, limits the fraction of nuclear states within the locking range $\Delta \delta_{\text {lock }}$ of the feedback mechanism, whereas higher driving power causes a power broadening of the dark-state resonance, reducing the strength of the feedback.

Results presented in Fig. 1 have important implications for quantum information processing. The full tenfold extension of the electron dephasing time requires a preparation duty cycle $T_{\mathrm{CPT}} /\left(T_{\mathrm{CPT}}+T_{R}\right) \gtrsim 40 \%$ [30]. Under the $1-\mathrm{kHz}$ repetition rate of our experimental sequence, more than 600 Ramsey sequences or other quantum operation of $1-\mu$ s duration could be performed following 
nuclear preparation. Moreover, the extension of $T_{2}^{*}$ well beyond the trion radiative lifetime of $\approx 0.7 \mathrm{~ns}$ nearly eliminates a key decoherence mechanism limiting the quality of spin-photon entanglement in QDs [26-28]. Finally, changing the lock point set by the two-photon detuning provides precise control on the electron splitting to within $1.5 \mathrm{MHz}$ over more than $3.5 \mathrm{GHz}$ [30], which can aid the generation of indistinguishable Raman photons for entanglement distribution between multiple QDs $[33,34]$.

The modification of the nuclear-spin distribution is a consequence of feedback-induced ensemble correlations, whose emergence is monitored using the electron FID profile as we vary the preparation time $T_{\mathrm{CPT}}$. Figure 2(a) shows the electron-spin coherence for $T_{\mathrm{CPT}}=0.5,1$, and $8 \mathrm{~ms}$ at a magnetic field of $6 \mathrm{~T}$. We fit the visibility with $C(\tau)=$ $A \exp \left[-\left(\tau / T_{2}^{*}\right)^{\alpha}\right]$, where throughout our analysis we describe the decay time and shape with $T_{2}^{*}$ and $\alpha$, respectively, thereby capturing the essential features linking the FID to the nuclear-spin distribution. Figures 2(b) and 2(c) present $T_{2}^{*}$ and $\alpha$ as a function of $T_{\mathrm{CPT}}$. As expected, $T_{2}^{*}$ increases with the preparation time to reach a steady-state value of $22 \mathrm{~ns}$ over a characteristic time $T_{p}=0.8 \pm 0.2 \mathrm{~ms}$. The exponent evolves nonmonotonically from $\alpha=2$, as

(a)

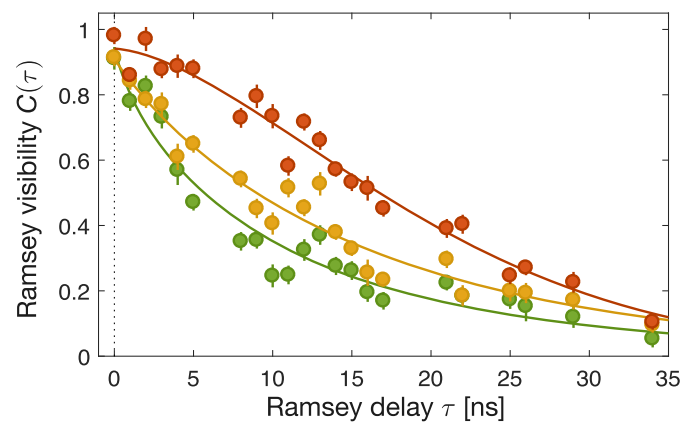

expected for the initial Gaussian state, dropping rapidly to values below 1 and later reaching a steady-state value of $\alpha=1.6$. This rich behavior suggests an interesting transient for the nuclear ensemble. Indeed, the Overhauser-field probability distribution $P\left(\delta_{n}\right)$ is given by the Fourier transform of the FID profile, provided the high-frequency nuclear noise is negligible. We achieve this limit with the 6-Texternal magnetic field at which fast dynamics of the nuclear ensemble due to quadrupolar interactions are suppressed $[23,24,30]$. Figures 2(d)-2(f) thus present the evolution of $P\left(\delta_{n}\right)$ corresponding to the data and the fits shown in Fig. 2(a). The decrease in the width of the distribution is accompanied by an evolution of its shape from resembling a Lorentzian, with significant spectral weight in its wings, to resembling a Gaussian. This behavior is a direct consequence of the CPT feedback mechanism, whose Overhauser-fielddependent gain imprints a transient distribution on the nuclear ensemble.

We can paint a simple picture of how the CPT-based feedback shepherds the nuclear spins into their steady-state distribution. The evolution of $P\left(\delta_{n}\right)$ is given by the spectral dependence of the average spin $\left\langle S_{x}\left(\delta_{n}\right)\right\rangle$; as shown in Fig. 1(a), there exist two Overhauser fields for which the (d)

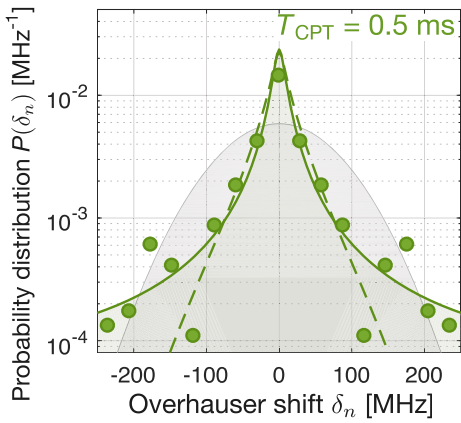

(e)

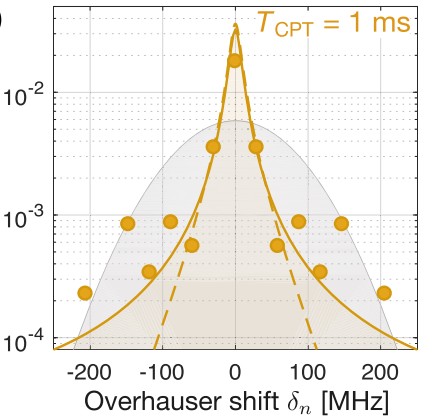

(b)

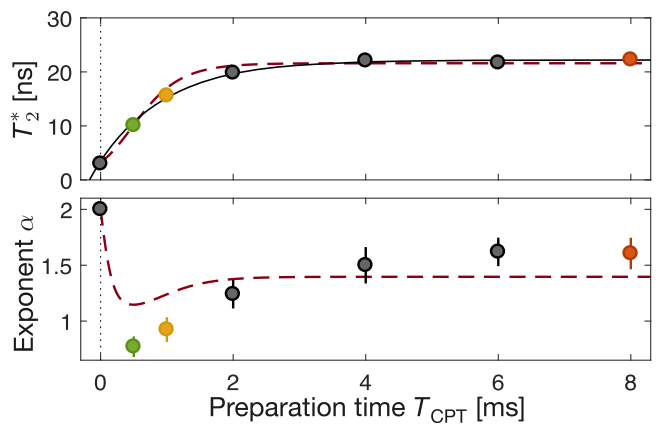

FIG. 2. Emergence of correlations within the nuclear ensemble. These data were taken at $6 \mathrm{~T}$ on $\mathrm{QD}_{B}$, whose $T_{2}^{*}$ is lower than that of $\mathrm{QD}_{A}$. (a) Ramsey visibility for a preparation time $T_{\mathrm{CPT}}=0.5 \mathrm{~ms}$ (green curve), $T_{\mathrm{CPT}}=1 \mathrm{~ms}$ (yellow curve), and $T_{\mathrm{CPT}}=8 \mathrm{~ms}$ (red curve). Solid curves are fitted with $C(\tau)=A \exp \left[-\left(\tau / T_{2}^{*}\right)^{\alpha}\right]$, where $A$ accounts for the power imbalance between the two rotation pulses. (b) Extracted $T_{2}^{*}$ and (c) $\alpha$ from the Ramsey visibility as a function of $T_{\mathrm{CPT}}$. The solid curve is a phenomenological exponential fit with characteristic time $T_{p}=0.8 \pm 0.2 \mathrm{~ms}$, while the dashed curves are numerical simulations. Error bars indicate a $67 \%$ confidence interval on the fitted values. (d)-(f) Fourier transforms of the electron FID (symbols) and their fits (solid curves) from (a) and simulated probability distributions of the Overhauser field (dashed curves) for $T_{\mathrm{CPT}}=0.5 \mathrm{~ms}$ (d), $T_{\mathrm{CPT}}=1 \mathrm{~ms}$ (e), and $T_{\mathrm{CPT}}=8 \mathrm{~ms}$ (f). Gray regions illustrate the Gaussian probability distribution of the unprepared nuclear ensemble. The discrepancy between the Fourier transform of the fits and experimental data below $3 \times 10^{-4} \mathrm{MHz}^{-1}$ is due to high-frequency noise in the experimental data. 
spin imbalance is maximal and, hence, the feedback is the strongest. At the early stages of preparation, the probability of finding the Overhauser field close to these maximum feedback points is depleted rapidly and redistributed towards the lock point. The wings of the distribution, where the feedback is weaker, are initially unaffected. This explains the fast reduction of the exponent $\alpha$ [Fig. 2(c)] and of the width of the central part of the distribution [Figs. 2(d) and 2(e)]. Then, as the preparation time is increased, only the wings of $P\left(\delta_{n}\right)$ can further contribute to narrowing [Fig. 2(f)] until $\alpha$ reaches its steady-state value.

Our measurements are consistent with theoretically anticipated values of $\alpha, T_{2}^{*}$, and $P\left(\delta_{n}\right)$ from a rate equation model [dashed curves in Figs. 2(b)-2(f)]. This model captures the effect of CPT on the electron-spin polarization, which in turn affects the average nuclear-spin polarization, causing an evolution of $P\left(\delta_{n}\right)$ under the Fokker-Planck formalism $[19,30,35]$. The feedback on the probability distribution is governed by the time derivative of the Overhauser field,

$$
\dot{\delta}_{n}=-\Gamma_{h}\left(\delta_{n}\right)\left[\delta_{n}-K\left\langle S_{x}\left(\delta_{n}\right)\right\rangle\right]-\Gamma_{d} \delta_{n} .
$$

Here, $\Gamma_{h}\left(\delta_{n}\right)$ is the optically assisted nuclear-flip rate whose spectral dependence follows the trion excited state population under CPT [yellow curve in Fig. 1(a)], and $\Gamma_{d}$ captures the dominant relaxation mechanism of the spin ensemble in the absence of optical excitation which is mediated here by the electron [30]. With a hyperfine-dependent gain factor $K$, the narrowing mechanism is driven by the ground-state spin polarization $\left\langle S_{x}\left(\delta_{n}\right)\right\rangle$, which provides the necessary directionality to spin flips to lock the nuclear ensemble. Quadrupolar effects are known to dominate spin flips in InGaAs QDs; the directionality of the feedback mechanism is therefore likely provided by a phenomenon known as spin dragging [14,15]. An approximate steady-state solution of the Fokker-Planck equation is an Overhauser-field distribution whose final variance is reduced by a factor $\alpha$ $K \Gamma_{h}(0) / \Delta_{\mathrm{CPT}} \Gamma_{d}$ from its initial thermal variance [30]. The model therefore predicts that the narrowing limit is determined by the interplay of the feedback strength $K / \Delta_{\mathrm{CPT}}$ and the strength of nuclear-spin diffusion $\Gamma_{d} / \Gamma_{h}(0)$.

In the absence of polarization diffusion out of the QD [36], the nuclear ensemble remains in its reduced-entropy state for a finite time before spin-spin interactions recover a thermal distribution over a correlation time $T_{c}$. This return towards thermal equilibrium can be monitored by introducing a wait time $T_{\text {relax }}$ between CPT preparation and Ramsey measurement, as shown in Fig. 3(a). Figure 3(b) presents the Ramsey visibility for $T_{\text {relax }}=0$ and $6 \mathrm{~ms}$ after a preparation time of $2 \mathrm{~ms}$. Figures 3(c) and 3(d) show the fitted $T_{2}^{*}$ and $\alpha$ as a function of $T_{\text {relax }}$. As $T_{\text {relax }}$ increases, the distribution tends to a thermal Gaussian shape ( $\alpha$ increases), and the enhancement of electron coherence is lost ( $T_{2}^{*}$ decreases). Assuming relaxation from a narrowed (a)
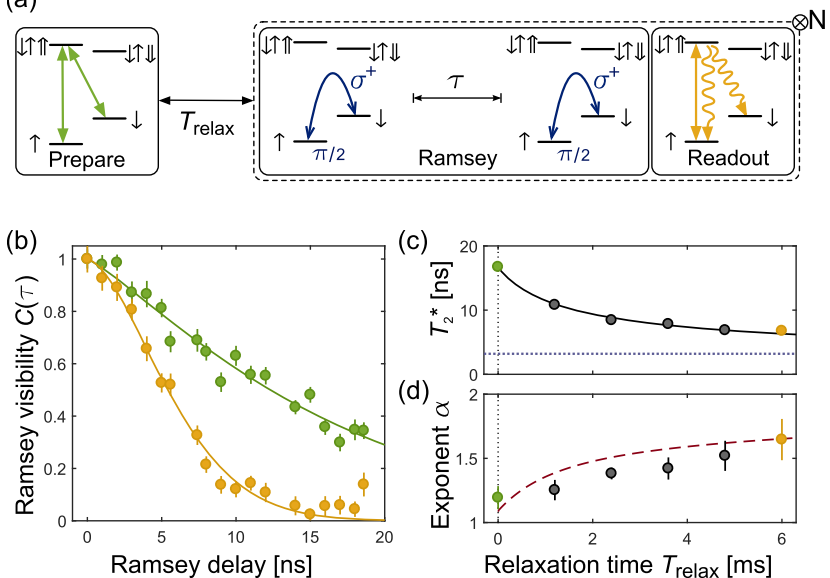

FIG. 3. Relaxation of the correlated nuclear ensemble. (a) Pulse sequence: A wait time $T_{\text {relax }}$ is introduced between the CPT preparation $\left(T_{\mathrm{CPT}}=2 \mathrm{~ms}\right)$ and the electron coherence measurement. (b) Electron FID profile for $T_{\text {relax }}=0$ (green curve) and $6 \mathrm{~ms}$ (yellow curve). The solid curves are fitted with $C(\tau)=A \exp \left[-\left(\tau / T_{2}^{*}\right)^{\alpha}\right]$. (c) $T_{2}^{*}$ and (d) $\alpha$ extracted from the fits. The solid curve is fitted with $T_{2}^{*}\left(T_{\text {relax }}\right)=$ $T_{2}^{*}(\infty) / \sqrt{1-B \exp \left(-2 T_{\text {relax }} / T_{c}\right)}$, where $T_{2}^{*}(\infty)$ is the unprepared $T_{2}^{*}$ [blue dashed line in (c)], and $B=1-\left[T_{2}^{*}(\infty) / T_{2}^{*}(0)\right]^{2}$, from which a correlation time $T_{c}=46.4 \pm 3.4 \mathrm{~ms}$ is obtained. The dashed curve in (d) is calculated from the Fokker-Planck equation assuming an initial distribution with $\alpha=1.1$ and $T_{2}^{*}(0)=17 \mathrm{~ns}$. Error bars indicate a $67 \%$ confidence interval on the fitted values.

Gaussian state whose variance evolves exponentially to its thermal value within a characteristic time $T_{c}$, the electron dephasing time follows the analytical expression $T_{2}^{*}\left(T_{\text {relax }}\right)=T_{2}^{*}(\infty) / \sqrt{1-B \exp \left(-2 T_{\text {relax }} / T_{c}\right)}$, the fitting function used in Fig. 3(c). While $T_{2}^{*}$ drops rapidly on a time scale of milliseconds, the extracted correlation time is $T_{c}=46.4 \pm 3.4 \mathrm{~ms}$. This correlation time is shorter than the estimated characteristic time of the polarization loss $T_{1}$ [30] but significantly longer than the nuclear coherence time $T_{2}$ [37]. The measurement of the nuclear relaxation allows us to fix the nuclear-spin relaxation rate $\Gamma_{d}+\Gamma_{h}(0)$ in our model, which, in addition to supporting our results in Fig. 2, reproduces the relaxation of $\alpha$ [Fig. 3(d)]. The overall consistency of the model with our data supports our interpretation that the order-of-magnitude improvement in $T_{2}^{*}$ is the best that can be achieved for our specific system with this technique.

We have shown that the interaction of a QD electron with its nuclei can be tailored to create reduced-entropy states of the nuclear ensemble. Such engineering of the electron-spin environment results in a tenfold increase in qubit coherence, which will directly improve the transfer of quantum information between a single spin and a single photon in QDs. The magnitude of this enhancement is dictated by the feedback strength set by the hyperfine interaction and by nuclear-spin diffusion. Furthermore, access to such a 
correlated spin ensemble sets the stage for investigations of quantum many-body physics in QDs, possibly leading to ensemble quantum memories $[38,39]$. Quantum correlations within the nuclei can be generated by the nonlinear interactions [40] provided in our current feedback mechanism by the strong dependence of the electron-spin polarization on the total nuclear spin around the CPT lock point. As proposed for directly driven electron-spin resonance [41], a polarized nuclear ensemble locked around a dark-state resonance together with coherent manipulation would lead to ensemble spin squeezing.

We acknowledge financial support from the European Research Council ERC Consolidator Grant Agreement No. 617985 and the EPSRC National Quantum Technologies Program NQIT EP/M013243/1. G.E-M. acknowledges financial support from NSERC. We thank M. J. Stanley and L. Huthmacher for fruitful discussions.

G. E.-M. and D. G. contributed equally to this work.

*ma424@cam.ac.uk

[1] E. Togan, Y. Chu, A. Imamoglu, and M. D. Lukin, Laser cooling and real-time measurement of the nuclear spin environment of a solid-state qubit, Nature (London) 478, 497 (2011).

[2] K. W. Murch, U. Vool, D. Zhou, S. J. Weber, S. M. Girvin, and I. Siddiqi, Cavity-Assisted Quantum Bath Engineering, Phys. Rev. Lett. 109, 183602 (2012).

[3] D. J. Reilly, J. M. Taylor, J. R. Petta, C. M. Marcus, M. P. Hanson, and A. C. Gossard, Suppressing spin qubit dephasing by nuclear state preparation, Science 321, 817 (2008).

[4] I. T. Vink, K. C. Nowack, F. H. L. Koppens, J. Danon, Y. V. Nazarov, and L. M. K. Vandersypen, Locking electron spins into magnetic resonance by electron-nuclear feedback, Nat. Phys. 5, 764 (2009).

[5] H. Bluhm, S. Foletti, D. Mahalu, V. Umansky, and A. Yacoby, Enhancing the Coherence of a Spin Qubit by Operating It as a Feedback Loop that Controls Its Nuclear Spin Bath, Phys. Rev. Lett. 105, 216803 (2010).

[6] X. Xu, W. Yao, B. Sun, D. G. Steel, A. S. Bracker, D. Gammon, and L. J. Sham, Optically controlled locking of the nuclear field via coherent dark-state spectroscopy, Nature (London) 459, 1105 (2009).

[7] B. Urbaszek, X. Marie, T. Amand, O. Krebs, P. Voisin, P. Maletinsky, A. Högele, and A. Imamoglu, Nuclear spin physics in quantum dots: An optical investigation, Rev. Mod. Phys. 85, 79 (2013).

[8] A. I. Tartakovskii et al., Nuclear Spin Switch in Semiconductor Quantum Dots, Phys. Rev. Lett. 98, 026806 (2007).

[9] B. Eble, O. Krebs, A. Lemaître, K. Kowalik, A. Kudelski, P. Voisin, B. Urbaszek, X. Marie, and T. Amand, Dynamic nuclear polarization of a single charge-tunable InAs/GaAs quantum dot, Phys. Rev. B 74, 081306(R) (2006).

[10] B. Urbaszek, P. F. Braun, T. Amand, O. Krebs, T. Belhadj, A. Lemaître, P. Voisin, and X. Marie, Efficient dynamical nuclear polarization in quantum dots: Temperature dependence, Phys. Rev. B 76, 201301(R) (2007).
[11] P. Maletinsky, A. Badolato, and A. Imamoglu, Dynamics of Quantum Dot Nuclear Spin Polarization Controlled by a Single Electron, Phys. Rev. Lett. 99, 056804 (2007).

[12] C. Latta, A. Srivastava, and A. Imamoglu, Hyperfine Interaction-Dominated Dynamics of Nuclear Spins in SelfAssembled InGaAs Quantum Dots, Phys. Rev. Lett. 107, 167401 (2011).

[13] A. Greilich, A. Shabaev, D. R. Yakovlev, A. L. Efros, I. A. Yugova, D. Reuter, A. D. Wieck, and M. Bayer Nucleiinduced frequency focusing of electron spin coherence, Science 317, 1896 (2007).

[14] A. Högele, M. Kroner, C. Latta, M. Claassen, I. Carusotto, C. Bulutay, and A. Imamoglu, Dynamic Nuclear Spin Polarization in the Resonant Laser Excitation of an InGaAs Quantum Dot, Phys. Rev. Lett. 108, 197403 (2012).

[15] C. Latta et al., Confluence of resonant laser excitation and bidirectional quantum-dot nuclear-spin polarization, Nat. Phys. 5, 758 (2009).

[16] T. D. Ladd, D. Press, K. De Greve, P. L. McMahon, B. Friess, C. Schneider, M. Kamp, S. Höfling, A. Forchel, and Y. Yamamoto, Pulsed Nuclear Pumping and Spin Diffusion in a Single Charged Quantum Dot, Phys. Rev. Lett. 105, 107401 (2010).

[17] D. Stepanenko, G. Burkard, G. Giedke, and A. Imamoglu, Enhancement of Electron Spin Coherence by Optical Preparation of Nuclear Spins, Phys. Rev. Lett. 96, 136401 (2006).

[18] M. Issler, E. M. Kessler, G. Giedke, S. Yelin, I. Cirac, M. D. Lukin, and A. Imamoglu, Nuclear Spin Cooling Using Overhauser-Field Selective Coherent Population Trapping, Phys. Rev. Lett. 105, 267202 (2010).

[19] A. R. Onur and C. H. van der Wal, Two-laser dynamic nuclear polarization with semiconductor electrons: Feedback, suppressed fluctuations, and bistability near twophoton resonance, arXiv:1409.7576.

[20] A. R. Onur, J. P. de Jong, D. O'Shea, D. Reuter, A. D. Wieck, and C.H. van der Wal, Stabilizing nuclear spins around semiconductor electrons via the interplay of optical coherent population trapping and dynamic nuclear polarization, Phys. Rev. B 93, 161204 (2016).

[21] B. Sun, C. M. E. Chow, D. G. Steel, A. S. Bracker, D. Gammon, and L. J. Sham, Persistent Narrowing of Nuclear-Spin Fluctuations in InAs Quantum Dots Using Laser Excitation, Phys. Rev. Lett. 108, 187401 (2012).

[22] C. M. Chow, A. M. Ross, D. Kim, D. Gammon, A. S. Bracker, L. J. Sham, and D. G. Steel, Nonlocal Nuclear Spin Quieting in Quantum Dot Molecules: Optically Induced Extended Two-Electron Spin Coherence Time, Phys. Rev. Lett. 117, 077403 (2016).

[23] A. Bechtold, D. Rauch, F. Li, T. Simmet, P.-L. Ardelt, A. Regler, K. Müller, N. A. Sinitsyn, and J. J. Finley, Threestage decoherence dynamics of an electron spin qubit in an optically active quantum dot, Nat. Phys. 11, 1005 (2015).

[24] R. Stockill, C. Le Gall, C. Matthiesen, L. Huthmacher, E. Clarke, M. Hugues, and M. Atatüre, Quantum dot spin coherence governed by a strained nuclear environment, Nat. Commun. 7, 12745 (2016).

[25] D. Press, K. De Greve, P. L. McMahon, T. D. Ladd, B. Friess, C. Schneider, M. Kamp, S. Höfling, A. Forchel, and Y. Yamamoto, Ultrafast optical spin echo in a single quantum dot, Nat. Photonics 4, 367 (2010). 
[26] K. De Greve et al. Quantum-dot spin-photon entanglement via frequency downconversion to telecom wavelength, Nature (London) 491, 421 (2012).

[27] W. B. Gao, P. Fallahi, E. Togan, J. Miguel-Sanchez, and A. Imamoglu, Observation of entanglement between a quantum dot spin and a single photon, Nature (London) 491, 426 (2012).

[28] J. R. Schaibley, A. P. Burgers, G. A. McCracken, L. M. Duan, P. R. Berman, D. G. Steel, A. S. Bracker, D. Gammon, and L.J. Sham, Demonstration of Quantum Entanglement between a Single Electron Spin Confined to an InAs Quantum Dot and a Photon, Phys. Rev. Lett. 110, 167401 (2013).

[29] Z. Sun, A. Delteil, S. Faelt, and A. Imamoğlu, Measurement of spin coherence using Raman scattering, Phys. Rev. B 93, 241302 (2016).

[30] See Supplemental Material at http://link.aps.org/ supplemental/10.1103/PhysRevLett.119.130503 for additional data, description, and analysis of results in this Letter. Supplemental Material includes Refs. [15,19,23,24,31].

[31] D. Press, T. D. Ladd, B. Zhang, and Y. Yamamoto, Complete quantum control of a single quantum dot spin using ultrafast optical pulses, Nature (London) 456, 218 (2008).

[32] D. Klauser, W. A. Coish, and D. Loss, Nuclear spin state narrowing via gate-controlled Rabi oscillations in a double quantum dot, Phys. Rev. B 73, 205302 (2006).

[33] A. Delteil, Z. Sun, W.-b. Gao, E. Togan, S. Faelt, and A. Imamoğlu, Generation of heralded entanglement between distant hole spins, Nat. Phys. 12, 218 (2015).
[34] R. Stockill, M. J. Stanley, L. Huthmacher, E. Clarke, M. Hugues, A. J. Miller, C. Matthiesen, C. Le Gall, and M. Atatüre, Phase-Tuned Entangled State Generation between Distant Spin Qubits, Phys. Rev. Lett. 119, 010503 (2017).

[35] J. Danon and Y. V. Nazarov, Nuclear Tuning and Detuning of the Electron Spin Resonance in a Quantum Dot: Theoretical Consideration, Phys. Rev. Lett. 100, 056603 (2008).

[36] P. Maletinsky, M. Kroner, and A. Imamoglu, Breakdown of the nuclear-spin-temperature approach in quantum-dot demagnetization experiments, Nat. Phys. 5, 407 (2009).

[37] G. Wüst, M. Munsch, F. Maier, A. V. Kuhlmann, A. Ludwig, A. D. Wieck, D. Loss, M. Poggio, and R. J. Warburton, Role of the electron spin in determining the coherence of the nuclear spins in a quantum dot, Nat. Nanotechnol. 11, 885 (2016).

[38] J. M. Taylor, C. M. Marcus, and M. D. Lukin, Long-Lived Memory for Mesoscopic Quantum Bits, Phys. Rev. Lett. 90, 206803 (2003).

[39] J. M. Taylor, A. Imamoglu, and M. D. Lukin, Controlling a Mesoscopic Spin Environment by Quantum Bit Manipulation, Phys. Rev. Lett. 91, 246802 (2003).

[40] M. Kitagawa and M. Ueda, Squeezed spin states, Phys. Rev. A 47, 5138 (1993).

[41] M. S. Rudner, L. M. K. Vandersypen, V. Vuletić, and L. S. Levitov, Generating Entanglement and Squeezed States of Nuclear Spins in Quantum Dots, Phys. Rev. Lett. 107, 206806 (2011). 